\title{
Inclusions and the Color of Obsidian
}

\author{
Andres Camargo ${ }^{1}$, Sarath Menon ${ }^{1}$, Claudia Luhrs ${ }^{1}$ and Raymond Mariella Jr. ${ }^{2}$ \\ ${ }^{1}$ Dept. of Mechanical and Aerospace Engineering, Naval Postgraduate School, Monterey, CA \\ ${ }^{2}$ Lawrence Livermore National Laboratory, Mail Stop L-222, PO Box 808, 7000 East Avenue, \\ Livermore, CA
}

Obsidian is a volcanic glass formed by the rapid quenching of the lava, is chemically similar to rhyolite or granite, and is composed mainly of silica with many other elements present in various oxide or silicate forms. Obsidian appears in a wide variety of colors though the jet-black variety is the most common. The color of obsidian depends on the wide variety of crystalline inclusions present in the glass with magnetite and some others providing a jet-black color $[1,2]$. When crushed into fine powder, the black obsidian appears nearly transparent white or with a slight gray tinge. This effect maybe due to the separation of the larger inclusions from the glassy powder produced when a piece of obsidian is crushed. Thus, when analyzing obsidian specimens, characterization of the inclusions in them is of prime importance.

We have employed obsidian as one of several targets in our studies on laser driven hydrothermal process (LDHP) used for the ablation of submerged samples. In order to evaluate the effects of the laser comminution process on the target sample, it is necessary to observe the morphological details of the inclusions in the obsidian glassy matrix before and after treatment. A sample of obsidian (Oregon, US) was examined by X-Ray Diffraction (XRD, Rigaku MiniFlex 600), Scanning Electron Microscopy (SEM, Zeiss Neon 40) and Transmission Electron Microscopy (TEM, FEI Osiris) including Energy Dispersive Spectroscopy (EDS) experiments with emphasis on examining the obsidian inclusions. For this study, several slices of the glass were carefully cut using a low speed saw with a diamond blade. A slice was polished following conventional metallographic sample preparation techniques and studied by optical and electron microscopy. For powder XRD studies, a slice of obsidian was crushed using a mortar and pestle. Some of the samples for SEM studies were given a $2.5 \mathrm{~nm}$ Pt-10Pd coating using a Ted Pella high resolution 208R sputter coater. Crushed samples were also dispersed in ethanol and placed in a holey carbon grid for TEM analysis. Optical microscopy of polished sections revealed that inclusions were randomly distributed within the specimen. Some of the larger inclusions presumably fell out during the polishing procedure leaving porosity behind [Figure 1(a)]. Often, stringers of inclusions are seen in the material as in [Fig. 1(b)]. XRD studies from the powder did not show any difference between the original slice before and after crushing [Figure 1(c)]. The material is composed of amorphous silica with a very small quantity of crystalline phase/s present in it. EDS analysis in the SEM showed that this particular piece of obsidian contained $\mathrm{Al}, \mathrm{Na}, \mathrm{Mg}, \mathrm{Ca}, \mathrm{Fe}$ and $\mathrm{Ti}$; on average, it was composed mostly of silica with roughly $13 \mathrm{wt} \%$ alumina and $1 \mathrm{wt} \%$ iron oxide apart from the other elements in smaller percentages. Secondary electron images and mapping from the crushed powder showed micron-sized Alrich, Fe-rich and, at times, Ca- rich particles [Figure 2]. EDS experiments in TEM showed that the amorphous silica particles in the crushed obsidian often contained very fine crystals $(<20 \mathrm{~nm})$ rich in Fe, $\mathrm{Ti}$ or $\mathrm{Na}$, suggesting the presence of various oxides and silicates embedded within the amorphous silica that constitute the bulk of obsidian. An example of such finding is shown in [Figure 3]. In the bulk pieces of obsidian, absorption of a wide range of wavelengths by the various inclusions of varying chemistry results in the black color of this sample. Once crushed, the small volume fraction of the inclusions in the powder mixture, mostly amorphous silica, leads to most of the light being scattered by the high volume fraction of the glass, supporting previous observations. The use of electron microscopy analysis was indispensable to detect all the inclusions (both at the nano and micron scales) and their components. This work was performed under award number HDTRA- 1514534 from DTRA. 


\section{References:}

1. http://volcano.oregonstate.edu/book/export/html/205

2. C. Ma, G. R. Rossman and J.A. Miller, The Canadian Mineralogist, Vol. 45 (2007) pp551-557.
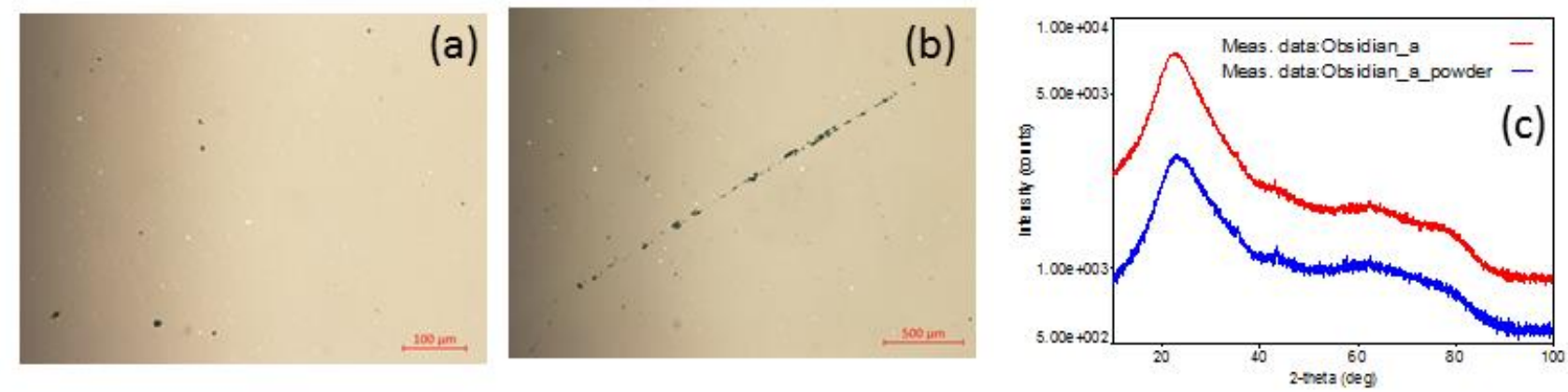

Figure 1. Optical micrographs of polished sections of obsidian showing the presence of inclusions in the structure are presented in (a) and (b). Notice the string of closely spaced inclusions in (b). (c) Shows the XRD patterns from bulk (RED) and powdered (BLUE) obsidian and the strong amorphous peaks indicate the glassy nature of obsidian.
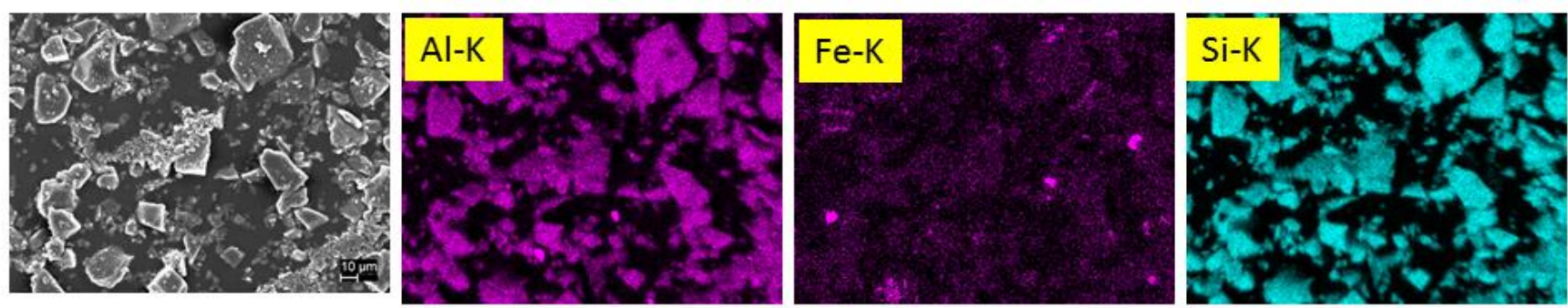

Figure 2. Secondary electron image and EDS maps from crushed obsidian showing the existence of micron-sized Fe-rich and Al-rich particles
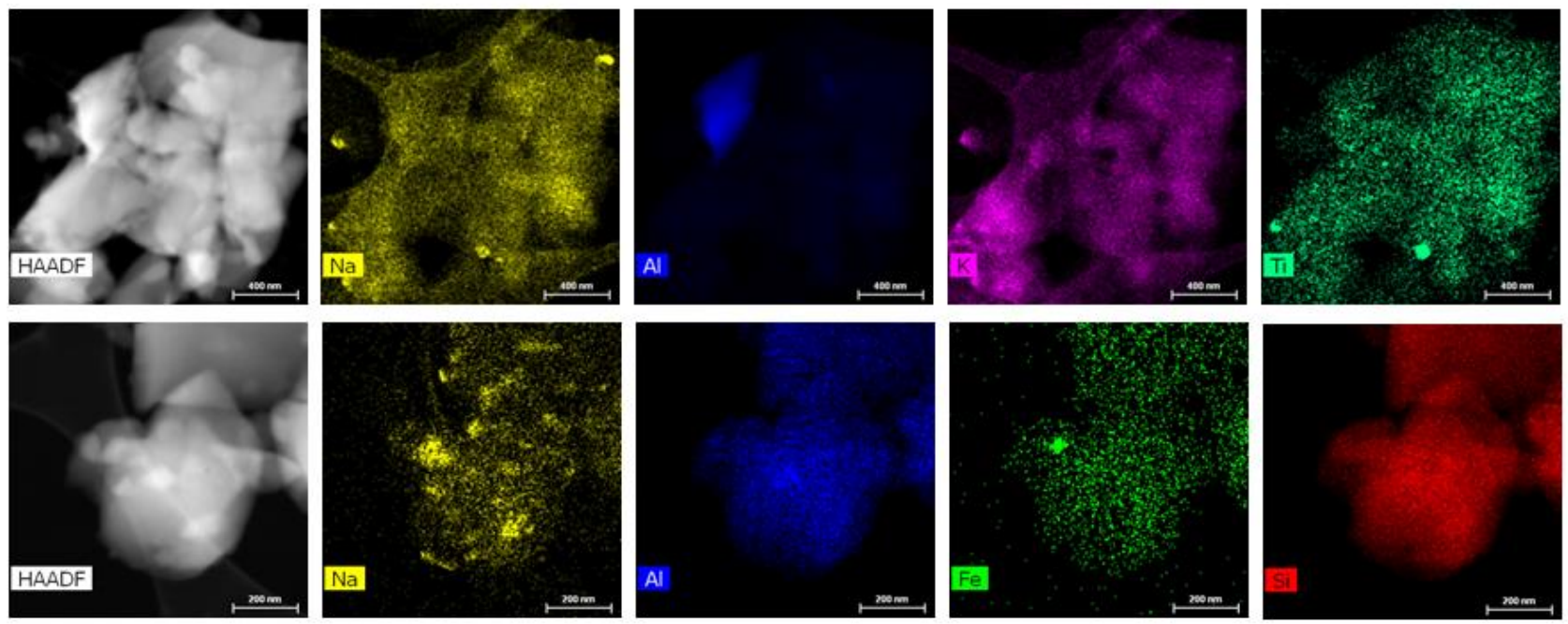

Figure 3. Examples of HAADF micrographs and EDS maps showing the presence on fine Fe-rich, Tirich or Na-rich inclusions in silica matrix of obsidian. 